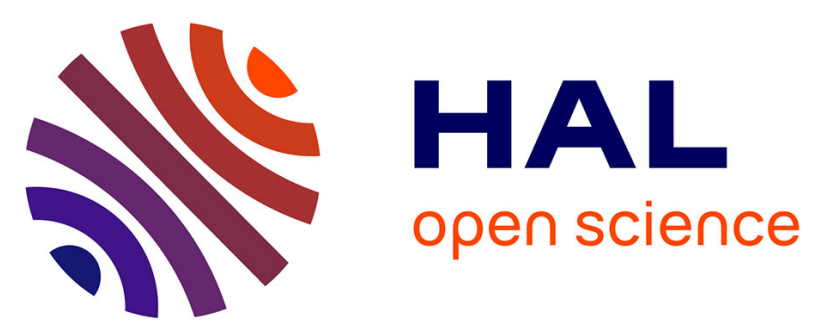

\title{
A multipurpose integrated preclinical device for the treatment of glioblastoma
}

Khaled Metwally, Chiara Bastiancich, Florian Correard, Anthony Novell, Samantha Fernandez, Benjamin Guillet, Benoit Larrat, Serge Mensah, Marie-Anne Estève, Anabela da Silva

\section{To cite this version:}

Khaled Metwally, Chiara Bastiancich, Florian Correard, Anthony Novell, Samantha Fernandez, et al.. A multipurpose integrated preclinical device for the treatment of glioblastoma. European Conferences on Biomedical Optics OSA/SPIE (ECBO 2021), Jun 2021, Munich (virtual), Germany. 10.1117/12.2614596 . hal-03313513

\section{HAL Id: hal-03313513 \\ https://hal.science/hal-03313513}

Submitted on 5 Aug 2021

HAL is a multi-disciplinary open access archive for the deposit and dissemination of scientific research documents, whether they are published or not. The documents may come from teaching and research institutions in France or abroad, or from public or private research centers.
L'archive ouverte pluridisciplinaire HAL, est destinée au dépôt et à la diffusion de documents scientifiques de niveau recherche, publiés ou non, émanant des établissements d'enseignement et de recherche français ou étrangers, des laboratoires publics ou privés. 


\title{
A multipurpose integrated preclinical device for the treatment of glioblastoma
}

\author{
Khaled Metwally, ${ }^{1,2, *}$ Chiara Bastiancich,, ${ }^{3, *}$ Florian Correard, ${ }^{3,4}$ Anthony Novell, ${ }^{5}$ \\ Samantha Fernandez, ${ }^{6}$ Benjamin Guillet,,${ }^{6,7}$ Benoit Larrat, ${ }^{8}$ Serge Mensah, ${ }^{2}$ \\ Marie-Anne Estève, ${ }^{3,4}$ Anabela Da Silva ${ }^{1, * *}$ \\ ${ }^{I}$ Aix Marseille Univ, CNRS, Centrale Marseille, Institut Fresnel, Marseille, France \\ ${ }^{2}$ Aix Marseille Univ, CNRS, Centrale Marseille, LMA, Marseille, France \\ ${ }^{3}$ Aix Marseille Univ, CNRS, UMR 7051 Institut Neurophysiopathologie, Marseille, France \\ ${ }^{4}$ APHM, Hôpital de la Timone, Service Pharmacie, Marseille, France \\ ${ }^{5}$ Université Paris-Saclay, CEA, CNRS, Inserm, BioMaps, Service Hospitalier Frédéric Joliot, Orsay, France \\ ${ }^{6}$ Aix-Marseille Univ, Centre Européen de Recherche en Imagerie Médicale (CERIMED), Marseille, France \\ ${ }^{7}$ Aix-Marseille Univ, INSERM, INRA, Center de Recherche en Cardiovasculaire et Nutrition (C2VN), Marseille, \\ France \\ ${ }^{8}$ Univ. Paris Saclay, CNRS, CEA, DRF/JOLIOT/NEUROSPIN/BAOBAB, Gif-sur-Yvette, France \\ ${ }^{*}$ Contributed equally to this work; ${ }^{* *}$ anabela.dasilva@fresnel.fr
}

\begin{abstract}
A multipurpose integrated preclinical device that combines Blood-Brain Barrier permeabilization, Photo-Thermal Therapy (PTT) with photoacoustic thermometry, was developed as a novel preclinical versatile research instrument for the development of new PTT for Glioblastoma multiforme. 이 2021 The Author(s)
\end{abstract}

\section{Introduction}

Glioblastoma multiforme (GBM) is one of the most common and aggressive malignant brain tumors in adults. As current therapies are ineffective, there is an urgent need to find novel and efficient therapeutic strategies.

One major limiting factor for brain tumors chemotherapies is the Blood-Brain Barrier (BBB), which prevents drugs from spreading from the vascular network to the central nervous system (CNS) limiting their penetration at the tumor site. To permeabilize the BBB and enhance drug delivery into the CNS, we use Focused Ultrasound Sonication (FUS) combined with intravascular microbubbles. Different in vitro and in vivo studies have demonstrated the interest of Photo-Thermal Therapy (PTT) in GBM treatment, chosen here as the main therapy. To improve heating performances and localization of the therapy, metallic nanoparticles (NPs) have emerged as potential photoabsorbing agents for PTT. Recently, laser-synthesized Titanium Nitride NPs (TiN NPs) have proven as new candidates [1,2], for which we have demonstrated in vitro (U87-MG GBM cell cultures) a low cytotoxicity combined with a good cellular uptake and PTT effect. To monitor the treatment, a photoacoustic (PA) thermoetry system was implemented for monitoring the temperature in real-time, in situ and in depth [3-6]. In the present study, we present this novel preclinical integrated device that allows performing on the same bench BBB opening with confocal FUS to improve local accumulation of NPs at the tumor site, highly localized PTT and non-invasive in situ PA thermometry.

The next section presents the fully integrated device developed. To demonstrate that the different features of this device are fully operational, we performed preliminary proof-of-concept in vivo studies that will be presented at the conference.

\section{Description of the integrated device}

A schema of the developed multi-functional setup is presented in Fig 1.

The device can be decomposed into three parts:

- The FUS/PTT/PA probe (Fig 1, Bottom) comprises a single-element focused transducer, presenting a hole in its center. The ultrasound transducer was then coupled to the head of the animal via the water balloon filled with deionized and degassed water to reach 7 to $10 \mathrm{~mm}$ thickness, depending on the targeted depth, and covered with degassed echographic gel. Electrical power sent to the transducer was monitored during the BBB opening session. The PA signal is collected through a digital oscilloscope. A hermetic glass window has been mounted and positioned on the top of the transducer mount in order to receive an optical fiber through which the light sources are delivered to the sample.

- The light delivery part includes two types of lasers, whose beams were piped through the same optical fiber: a CW laser diode for the PTT $(830 \mathrm{~nm}, 2 \mathrm{~W})$, controlled in current and temperature and a pulsed laser for the PA 


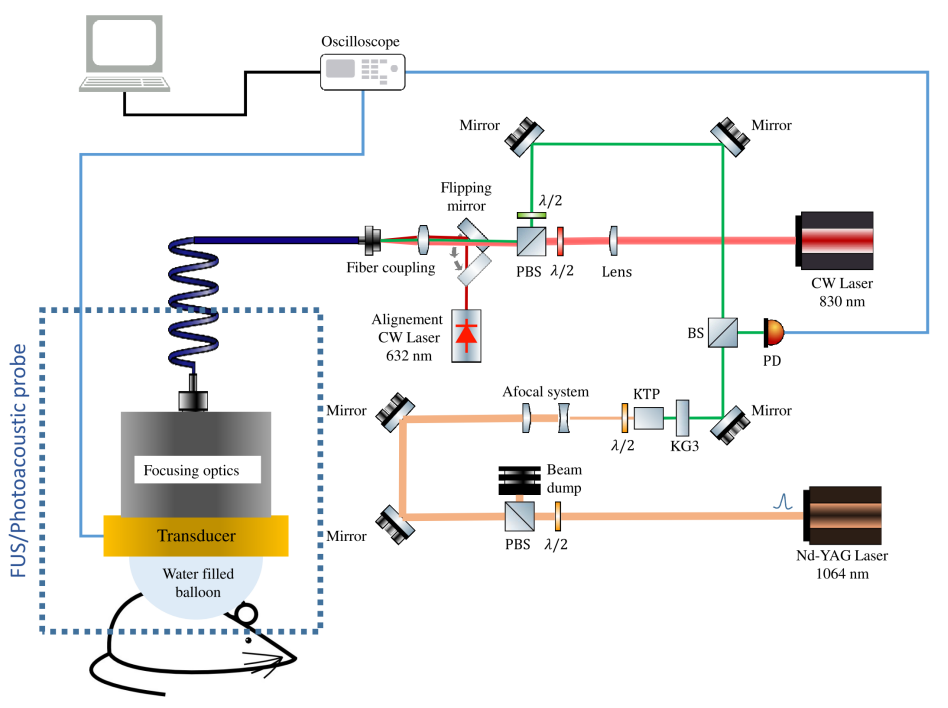

Fig. 1: Schema of the integrated experimental setup (PBS: Polarizing beam splitter; BS: Beam sampler; KTP: Frequency doubling KTP crystal; KG3: Filter glass (Bandpass: 315 - $710 \mathrm{~nm}$ ); PD: Si Photodetector (Detector, $200-1100 \mathrm{~nm}) ; \lambda / 2$ : Achromatic half-wave plate).

(Nd:Yag, $1064 \mathrm{~nm}, 330 \mathrm{~mJ}, 5 \mathrm{~ns}, 10 \mathrm{~Hz}$ ). The pulsed laser was frequency doubled with a KTP crystal at $532 \mathrm{~nm}$.

- A platform comprising a small animal holder, a transducer holder mounted on fully programmable 3D scanning stages and a programmable ultrasound single channel amplifier. Hot water is conveyed to the animal bed via plastic tubes to keep the body temperature of the animal stable during the therapy. The temperature of the circulating water is stabilized.

The different acoustic and optical emissions and data acquisition were controlled with a computer. The proposed FUS/PTT protocol includes three consecutive steps: i) systemic administration of efficient photoabsorbers (NPs or others); ii) injection of intravascular microbubbles followed by the application of FUS, by using the transducer in its active mode, with calibrated sonication parameters allowing BBB opening; iii) application of the PTT with PA temperature monitoring with a calibrated optical irradiation dose inducing tumor cell death.

\section{PhotoThermal Therapy and temperature monitoring with PhotoAcoustics: Preliminary tests on subcu- taneous GBM model}

The laser parameters selected for the in vivo studies (laser wavelength, power and heating duration) were based on values taken from literature [2] and the efficacy results obtained in vitro on U87-MG spheroids using TiN NP [1]. Within the linear approximation, for water-based and fatty tissues, the photoacoustic signal can be directly related to temperature [4,5,7]: $\Delta P / P_{o}=\alpha \Delta T$, where $P_{o}$ is the amplitude of the PA signal at a reference temperature $T_{o}$ and $\alpha$ is a calibration factor to be evaluated for the probed sample.

Here is reported a preliminary illustrative experiment of PTT and PA thermometry, performed on mice bearing subcutaneous tumors $(n=6)$. The group was devided into two groups, one $(n=3)$ without NPs, the second $(n=3)$ with NPs. The temperature variations during PTT $(\Delta \mathrm{T})$, estimated by considering a previously determined calibration factor are plotted in Fig. 2.
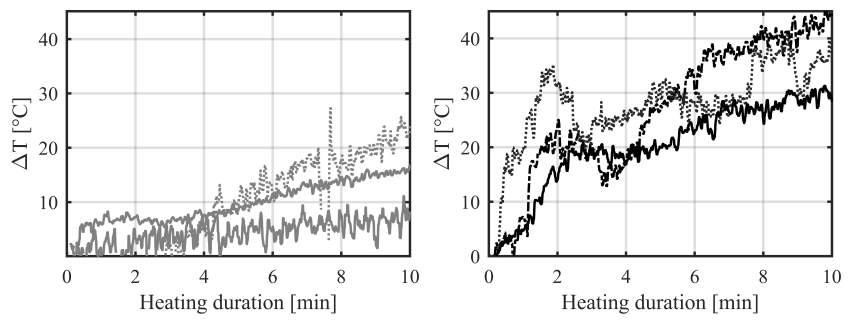

Fig. 2: Evolution of the estimated temperature rise during the PTT on mice from group without NPs (Left) and with NPs (Right). 


\section{Conclusion}

An original multipurpose integrated setup has been developed for efficient PTT of the glioblastoma in small animals [8]. It allows combining for the first time i) BBB opening by FUS for an increased efficiency in the targeted drug delivery to the brain; ii) PTT and iii) in situ temperature monitoring with PA. Sub-cutaneous and transcranial preliminary experiments were performed and demonstrated that the whole device is functional. Through skull PTT has also been shown feasible.

\section{References}

1. A. A. Popov, G. Tselikov, N. Dumas, C. Berard, K. Metwally, N. Jones, A. Al-Kattan, B. Larrat, D. Braguer, S. Mensah, A. Da Silva, M.-A. Estève, and A. V. Kabashin, "Laser-synthesized TiN nanoparticles as promising plasmonic alternative for biomedical applications," Scientific Reports 9, p. 1194, 2019.

2. C. Bastiancich, A. Da Silva, and M.-A. Estève, "Photothermal therapy for the treatment of Glioblastoma: potential and preclinical challenges ," Frontiers in Oncology 10, p. 610356, Jan 2020.

3. I. Larina, K. Larin, and R. Esenaliev, "Real-time optoacoustic monitoring of temperature in tissues," Journal of Physics D-Applied Physics 38, pp. 2633-2639, AUG 72005.

4. J. Shah, S. Park, S. Aglyamov, T. Larson, L. Ma, K. Sokolov, K. Johnston, T. Milner, and S. Y. Emelianov, "Photoacoustic imaging and temperature measurement for photothermal cancer therapy," Journal of Biomedical Optics 13, MAY-JUN 2008.

5. S.-H. Wang, C.-W. Wei, S.-H. Jee, and P.-C. Li, "Photoacoustic temperature measurements for monitoring of thermal therapy," in Photons Plus Ultrasound: Imaging And Sensing 2009, Oraevsky, AA and Wang, LV, ed., Proceedings of SPIE 7177, SPIE; Fairway Med Technol, Inc, 2009. Conference on Photons Plus Ultrasound - Imaging and Sensing 2009, San Jose, CA, JAN 25-28, 2009.

6. S. M. Nikitin, T. D. Khokhlova, and I. M. Pelivanov, "Temperature dependence of the optoacoustic transformation efficiency in ex vivo tissues for application in monitoring thermal therapies," Journal of Biomedical Optics 17, JUN 2012.

7. H. Ke, S. Tai, and L. Wang, "Photoacoustic thermography of tissue," Journal of Biomedical Optics 19, 2014.

8. K. Metwally, C. Bastiancich, F. Correard, A. Novell, S. Fernandez, B. Guillet, B. Larrat, S. Mensah, M.-A. Estève, and A. Da Silva, "Development of a multi-functional preclinical device for the treatment of glioblastoma," Biomedical Optics Express, p., under review. 